\title{
Proposal for a hardness measurement technique without indentor by gas-cluster-beam bombardment
}

\author{
Zinetulla Insepov, Rafael Manory, Jiro Matsuo, and Isao Yamada \\ Ion Beam Engineering Experimental Laboratory, Kyoto University, Sakyo-ku Kyoto 606 8501, Japan
}

(Received 23 March 1998; revised manuscript received 4 October 1999)

\begin{abstract}
Large gas-cluster-ion bombardment has been shown to be a unique tool for generating a variety of bombarding effects over a broad range of acceleration energies. A hardness measurement technique is proposed in this paper based on the use of the effect of crater formation by large gas-cluster beams. The cluster impact leaves a hemispherical crater on a surface, the size of which varies with surface hardness and cluster parameters (which can be predetermined). As shown in this paper, the crater depth $h$ (or diameter $d$ ) and Brinell hardness $B$ are correlated through the formula $h \sim(E / B)^{1 / 3}$, where $E$ is the cluster acceleration energy. The material hardness, binding energy, and the crater size have also been correlated with the sputtering yield $Y$, and hence this correlation can also be experimentally applied for measuring hardness. The proposed method is based entirely on surface effects which depend only on the surface material and not on the substrate and therefore should be particularly suitable for measuring hardness of thin deposited films. This technique also eliminates the need for indentors that are harder than the material measured.
\end{abstract}

\section{INTRODUCTION}

Clusters, or assemblies of atoms, are aggregates which can consist of many weakly bound atoms or molecules. Beams of large clusters can be generated in supersonic expansions of gas into vacuum through a nozzle. Electronic ionization of the neutral clusters and their electrostatic acceleration lead to the formation of energetic cluster ion beams. These beams can be used to bombard a target placed in the same vacuum chamber, and can simultaneously deliver large numbers of cluster atoms at low energy per atom while simultaneously getting sputtering yields from the target many orders of magnitude higher than that of a monomer ion irradiation. This unique feature of cluster ion beams has already been used for surface smoothing, shallow implantation, and other surface effects which occur when surfaces are irradiated with cluster ions, at ion doses of $10^{13}-10^{15}$ ions $/ \mathrm{cm}^{2} .{ }^{1-7}$

\section{A. Crater formation with hypersonic velocity impacts}

The phenomenon of crater formation is well known in so-called hypersonic velocity (or hypervelocity) impacts of macroscopic bodies on a solid surface at velocities in the range $\nu_{p} / c \geqslant 1$, where $\nu_{p}$ and $c$ are the projectile and sound velocities (for a target material), respectively. Crater formation at hypervelocity impacts of macroscopic projectiles on metal surfaces was studied in Refs. 8-10. It was shown that at a hypervelocity impact, for $\nu_{p}<10 \mathrm{~km} / \mathrm{s}$, the crater depth fits well the empirical formula (in CGS units in the original [a misprint of the original formula was corrected in Eq. (1)])

$$
h / D_{p}=\left(\frac{12 \times 10^{-9}(E / B)}{2 \pi D_{p}^{3}}\right)^{1 / 3},
$$

where $h$ is the crater depth, $D_{p}$ is the projectile diameter in $\mathrm{cm}, E$ is the projectile energy in erg, and $B$ is the standard Brinell hardness number in $\mathrm{kg} / \mathrm{mm}^{2}$. The shape of the macroscopic craters has been obtained to be a hemispherical. ${ }^{8-10}$
As it is seen from this formula, the crater depth $h$ does not depend on the projectile momentum, but on the impact energy $E$ only. For higher $\nu_{p}$ values, the projectile momentum contributes more to the crater shape, and the exponent is slightly smaller. ${ }^{9}$

According to this empirical formula, the projectile energy $E$ divided by crater volume $V_{\mathrm{cr}} \sim h^{3}$ should be linearly proportional to the Brinell hardness of the target material: $E / V_{\text {cr }} \sim B$ for a hypervelocity impact with the velocity less than about $10 \mathrm{~km} / \mathrm{s}$. This correlation has been experimentally confirmed for a variety of metals which includes lead, aluminum, copper, bronze, brass, steel, titanium for projectile masses ranging from $10^{-11}$ to $10 \mathrm{gm}$ (i.e., 12 orders of magnitude) for velocities up to about $15 \mathrm{~km} / \mathrm{s} .{ }^{9}$ The measured crater dimensions were the depth and the radius which were obtained to be about equal.

One of the most significant effects of bombardment by heavy monomer, molecular, and cluster ion beams, with a total energy of about $10-500 \mathrm{keV}$, is the formation of atomic scale craters, with diameters of about $\sim 10-100 \AA .^{1-7,11-14}$

Merkle and Jäger ${ }^{11}$ observed crater formation by TEM on $\mathrm{Au}$ foils due to $10-500 \mathrm{keV}$ irradiation by $\mathrm{Bi}$ and $\mathrm{Bi}_{2}$ ions. Thompson and Johar ${ }^{12}$ proposed the existence of an energy threshold for crater formation with heavy monomer ion impacts above which this phenomenon can occur. The threshold energies given in Refs. 12 are for $\mathrm{Ag}, 3.04 \mathrm{eV}$, for $\mathrm{Au}$, $3.78 \mathrm{eV}$, and for Pt, $5.95 \mathrm{eV}$. These data are well correlated with the binding energies of these metals. ${ }^{13}$ This correlation, which was observed experimentally, also shows that the dynamics of crater formation for heavy ion impact is controlled by the total ion energy released at impact rather than by the ion momentum.

Formula (1) was originally obtained for large craters, with diameters of about $1 \mathrm{~cm}$, created with macroscopic projectiles having hypersonic velocities. It is not directly applicable for microscopic small craters created by single heavy ions because the ions lose their energy in collisions with target 
electrons. Nevertheless, there is a similarity in the physics of crater formation due to a single heavy ion bombardment creating a track and a macroscopic body impact forming a crater.

So far it has not been shown that this formula is valid for small craters made by accelerated cluster impacts. However, in Ref. 14 this formula was used to estimate crater depths to be in the order of 20-300 $\AA$ when $\mathrm{Cu}$ and Ti surfaces were eroded with $\mathrm{CO}_{2}$ and Cs clusters $(n=100-1000)$, with energies of $1-500 \mathrm{keV}$.

For the ion and surface engineering communities, a direct relationship between physical properties of different surfaces and crater dimensions, sputtering yield, and erosion rate would have a distinctive advantage over the present state of the art: If a correlation such as Eq. (1) could be confirmed, surface hardness could practically be obtained from other data measured routinely in cluster ion beam experiments, without performing the actual hardness measurement on another instrument.

\section{B. Known difficulties in surface hardness measurement}

Hardness is commonly described as the resistance of the material to penetration of an indentor. Teter and Hemley ${ }^{15,16}$ refer to the known difficulties of hardness testing and interpretation by a quote from a book published in 1934: ${ }^{17}$ "Hardness, like the storminess of seas, is easily appreciated but not readily measured.',

Until the relatively recent introduction of the nanohardness method ${ }^{18}$ in which the hardness is obtained continuously in a load-unload cycle, hardness evaluation was performed by methods in which an indentor penetrates the surface at a known load. Hardness values are obtained by measuring the surface deformation after removing the indentor. The hardness is determined either by measuring the shape or the depth of the residual indentation.

Hardness values which can be directly correlated with physical properties, such as tensile strength, are obtained in techniques such as Brinell, Vickers, or Knoop in which the surface geometry of the residual indentation is measured by optical methods. The basic formula for hardness is $P / A$, where $P$ is the load and $A$ the surface area of the indentation. For Brinell hardness number (BHN), the formula is

$$
B=\frac{2 P}{\pi D\left(D-\sqrt{\left(D^{2}\right.}-d^{2}\right)},
$$

where $D$ is the indentor diameter and $d$ is the diameter of the residual indentation. ${ }^{19}$ Hardness values thus obtained can be directly expressed in Pascals, as the load is known and the surface area of the indentation is obtained from its diameter.

A significant problem encountered in hardness measurements is the measurement of treated surfaces and coatings. The indentation trace in this case is significantly influenced by an elastic contribution from the substrate material. This effect is reduced with increasing coating thickness, but it can vary significantly with substrate. Vickers hardness values of TiN films deposited simultaneously on various substrates ${ }^{20}$ were found to vary at low loads between $25000 \mathrm{MPa}$ on hard steel and $4000 \mathrm{MPa}$ on Al. The fact that the same type of thin film can present different values on different substrates means that in most cases hardness values can only be used for comparison and that they are not absolute. This also leads to some doubts regarding the accuracy of much of the data currently at hand in the literature.

A significant improvement in the field of hardness measurements was the development of the nanohardness (or ultramicrohardness) testing method in which the load vs penetration depth curve is recorded during penetration of a conical indentor into the surface at very low load ${ }^{18}$ and data from the near surface region is collected as a function of depth and thus it was believed to be less influenced by the substrate. Nevertheless, problems in the interpretation of results obtained from hard deposited coatings are still common, and the technique is not free from substrate effects.

It seems that at present there is no method which is reliable for absolute hardness values, particularly for thin film coatings, confirming the abovementioned view expressed in Ref. 17. This paper aims to propose a solution to this problem which has been made feasible with recent developments in surface microscopy [surface tunneling microscopy (STM) and atomic force microscopy (AFM)] and in cluster ion beam technology.

The structure of the paper is as follows. In Sec. II, shockwave generation with large $\mathrm{Ar}$ gas cluster impact on a $\mathrm{Cu}(100)$ surface is presented based on molecular dynamics (MD) simulation. Section III presents a theoretical estimation of crater dimensions, based on hydrodynamics and MD results, as well as on experimental crater data obtained with large gas cluster ion impacts. In Sec. IV, another type of experimental data, namely, sputtering yield is considered as an alternative for estimation of crater size. The technique of sub-nanohardness measurement which can be performed using cluster ion bombardment and which should be free of substrate effects, is proposed in Sec. V.

\section{SHOCK WAVE GENERATION WITH CLUSTER ION IMPACTS}

In various experimental STM observations ${ }^{21,22}$ a hemispherical crater was obtained on the surface after cluster ion bombardment. Theoretical studies of heavy single ion impacts based on a shock wave viewpoint were performed based on a thermal diffusion equation or on Hugoniot's relation. ${ }^{23-27}$ These works were successful in obtaining quantitative results regarding the physics of crater formation as well as estimates of sputtering yield, without dealing with the dynamics of shock waves or crater formation. As well, no relationship between ion energy and crater characteristics was obtained in these papers. ${ }^{23-27}$

Large cluster ion impacts have been studied by a molecular dynamics method in Refs. 2-7. MD has also been used to calculate temperature, pressure and energy of planar (onedimensional) steady-state shock waves, ${ }^{28-31}$ to determine the velocity of a surface shock wave due to ion impact, ${ }^{32}$ to simulate a shock wave generation within a cluster, ${ }^{33}$ and to study cluster impacts. ${ }^{34}$

Because of the inevitable nonsteady state character of the ion impact, it is very difficult to perform computer simulation of shock waves generated at such an impact. Webb and Harrison $^{32}$ were the first to calculate by MD the velocity of the shock wave generated with $5 \mathrm{Kev} \mathrm{Ar}^{+}$ion impact on a 
$\mathrm{Cu}$ surface, to be $17.6 \mathrm{~km} / \mathrm{s}$. Hypervelocity Ar cluster impact on a rigid target surface and generation of a shock wave within a cluster have been modeled by MD in Ref. 33. A two-dimensional (2D) MD method was used in our previous work $^{34}$ where shock wave generation was studied at an $\mathrm{Ar}$ cluster impact on a movable atomistic surface. As we have shown, when a large gas cluster hits a solid surface with hypersonic velocity, it penetrates into the target as a whole to a depth which depends on cluster energy. A strong pressure wave of about $100 \mathrm{kbar}$ is generated due to impact.

In the present paper, the dynamics of a hypervelocity Ar cluster impact on a $\mathrm{Cu}(001)$ surface is analyzed with a threedimensional MD method. Clusters were formed from Ar atoms interacting via Buckingham potential and an embedded atom method (EAM) potential was used to describe interactions between $\mathrm{Cu}$ atoms. The collisions of $\operatorname{Ar}_{n}(n$ =236-736) clusters with a $\mathrm{Cu}(100)$ surface were modeled. The total number of target atoms was about 77000 for energy of $6-13 \mathrm{keV}$, and about $10^{5}$ for energy of about $20 \mathrm{keV}$.

In our previous paper, ${ }^{35}$ a hybrid molecular dynamics (HMD) method was proposed, which combines conventional atomistic MD for the central cluster collisional zone, with a continuum mechanics representation for the rest of the system. This approach significantly reduces the system size and can keep the accuracy of the energy flow through the system boundaries. According to this technique, the response of the continuum part to the atomistic MD part can be represented by two components-one which is determined by forces calculated from a stress tensor and depends on the magnitude of deformation of the boundary layers, and the second which controls the energy balance and is introduced by energy absorbing walls, which were simulated by thermal diffusion equations. In the present paper the same boundary conditions were used, as in Ref. 35 ("thermal" boundaries).

The basic MD cell was divided into spherical layers of width $d r$ and the local target variables such as temperature, pressure, energy, and the velocity of moving matter (mass velocity) within a spherical layer were calculated with a certain time step, for the whole computation time. Local target temperatures were obtained from the equipartition theorem by deducting atomic kinetic energies from the average kinetic energy for the given spherical layer and local pressures were calculated from virial formula. ${ }^{7,28,29,36}$

A shock wave front in an ideal nonviscous and a nonthermal conductive gas is a zero-thickness surface which moves with hypersonic velocity. In a real solid it has a certain thickness defined by the real material viscosity and thermal conductivity. ${ }^{37}$ At a shock front, the local temperature, pressure, and energy abruptly acquire an increase from their equilibrium values before the front, e.g., room temperature and zero pressure, to much higher values behind the front. In a classical (macroscopic) shock, the pressure, volume (or density), and temperature in front of and behind the wave are related through a simple formula known as Hugoniot's relation which represents mass, momentum, and energy conservation laws. ${ }^{37}$

The atomic scale shock wave emerging from the cluster impact was obtained as a steep increase of radial and transversal kinetic energies of the target atoms according to the technique described above for which a spherical layer thickness $d r=3 \AA$ was used, as in Refs. 28 and 29. The front of a)

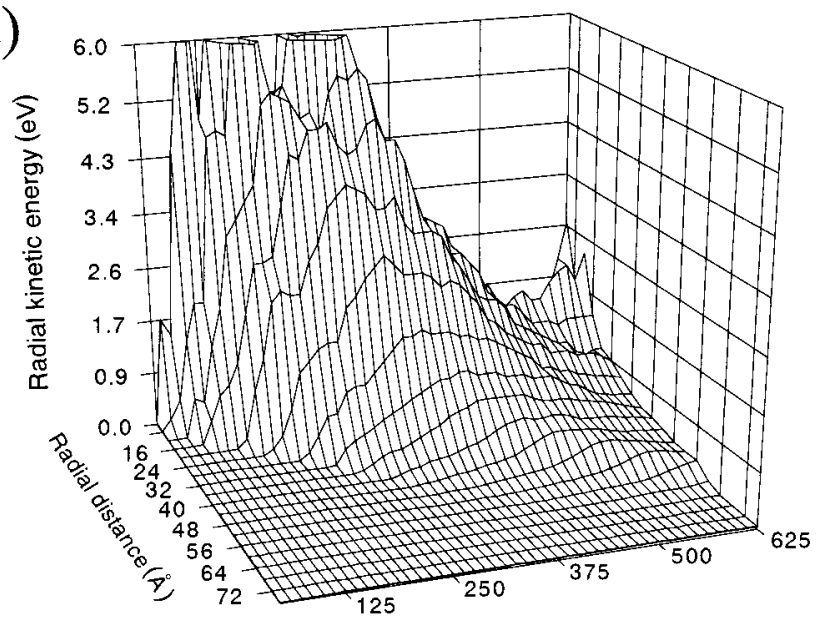

time $(f s)$

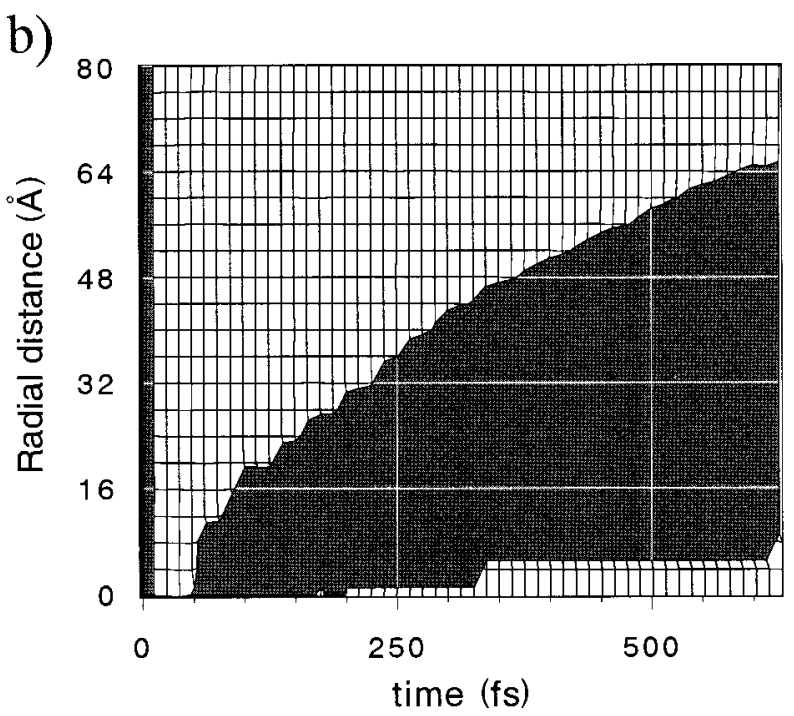

FIG. 1. MD calculation of a shock wave generated at a $\operatorname{Ar}_{370}$ cluster ion impact on a $\mathrm{Cu}(100)$ surface, with energy of $27 \mathrm{eV}$ atom (the total energy $10 \mathrm{keV}$ ). (a) shows space and time dependence of kinetic energy within a spherical layer of $3 \AA$ thickness at a radial distance $r$ from the impact mark and at a time $t$ from the beginning of the impact. The bottom figure shows a top view of (a) with the $z$ axis cut at a temperature higher than room temperature thus showing a shock wave front trajectory (b). The black areas are the states behind the shock front, and the white areas in front of the shock.

this rise was considered as a shock wave front. This definition of a shock wave front was used in Refs. 30 and 31 for a planar shock.

The time and space dependence of radial kinetic energy for an $\mathrm{Ar}_{370}$ cluster impact with energy of $27 \mathrm{eV} /$ atom on a $\mathrm{Cu}(100)$ surface with a total cluster energy of $10 \mathrm{keV}$ is shown in Fig. 1(a). Figure 1(b) shows the top view of Fig. 1(a) with a cut of the $z$ axis at a certain level higher than room temperature, thus representing a trajectory of the pulse. Black areas in this figure correspond to temperatures higher than room temperature (behind the shock wave front) and white areas show equilibrium states ahead of the front. As can be seen from this figure, a strong pulse, or shock wave, propagates into the solid. The velocity of the shock front could be obtained from this figure to be about $10 \mathrm{~km} / \mathrm{s}$. The shock wave penetrates to a distance of about $65 \AA$ within a 


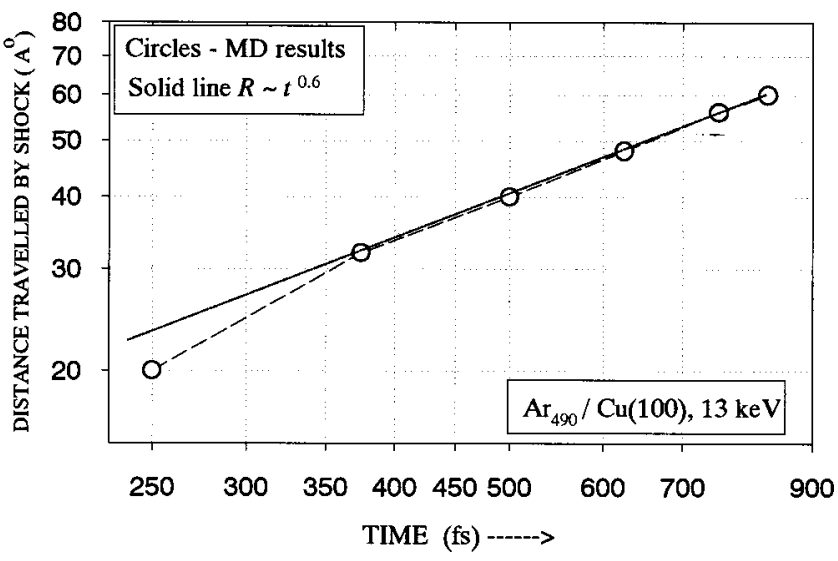

FIG. 2. Distance traveled by shock front calculated by MD for $\mathrm{Ar}_{490}$ cluster ion impact with total energy of $13 \mathrm{keV}$ on a $\mathrm{Cu}(100)$ surface (circles), the straight line corresponds to the $t^{0.6}$ power law.

time interval of $0.5 \mathrm{ps}$, while the cluster itself penetrates to a lesser distance of about $10 \AA$. Figure 1 shows that the velocity of the shock wave front rapidly decreases after about 0.6 ps and the shock wave almost disappears by this distance.

Figure 2 shows the radial distances $R(t)$ traveled by a shock front for a wave generated by an $\mathrm{Ar}_{490}$ cluster ion impact with total energy of $13 \mathrm{keV}$ on a $\mathrm{Cu}(100)$ surface. Fitting this MD result by the time dependence $R(t) \sim t^{\alpha}$ gives $\alpha \approx 0.6$. The straight solid line in Fig. 2 corresponds to this dependence. As can be seen from this figure, the dependence fits well for the latest time interval of the impact, after about 375 fs. The same value of $\alpha$ was obtained for impacts at 6,10 , and $19.9 \mathrm{keV}$. This time dependence of the distance traveled by a shock front could be easily measured experimentally. The calculated value is very close to the value 0.61 measured in a laser ablation experiment. ${ }^{38}$

\section{CRATER FORMATION}

The depth of a crater formed by cluster ion bombardment of a solid surface can be roughly estimated from the mass, momentum and energy conservation laws assuming that the impact generates a shock wave

$$
\begin{gathered}
E_{0}=E_{i}+E_{\mathrm{hyd}}, \\
E_{i}=\frac{1}{2} \overline{P_{H}} V, \\
\overline{P_{H}}=P_{c}+P_{\mathrm{th}},
\end{gathered}
$$

where $E_{0}$ is the total cluster ion energy, $E_{i}$ and $E_{\text {hyd }}$ are, respectively, the internal energy of a compressed area and the energy of a radial hydrodynamic motion of the compressed material encompassed by shock compression, as a whole. $\overline{P_{H}}$ is the Hugoniot's pressure, $V$ is the crater volume, $P_{c}$ and $P_{\text {th }}$ are the cold and thermal pressure components, respectively.

For weak shock waves, with $P_{H}$ well below $10^{4} \mathrm{MPa}$, the two energy components on the right side of Eq. (2a) are equal, and $P_{\text {th }}$ in Eq. (2c) can always be neglected in comparison with $P_{c}$. For example, at $30 \%$ compression the total pressure behind the shock wave for $\mathrm{Pb}$ has the following

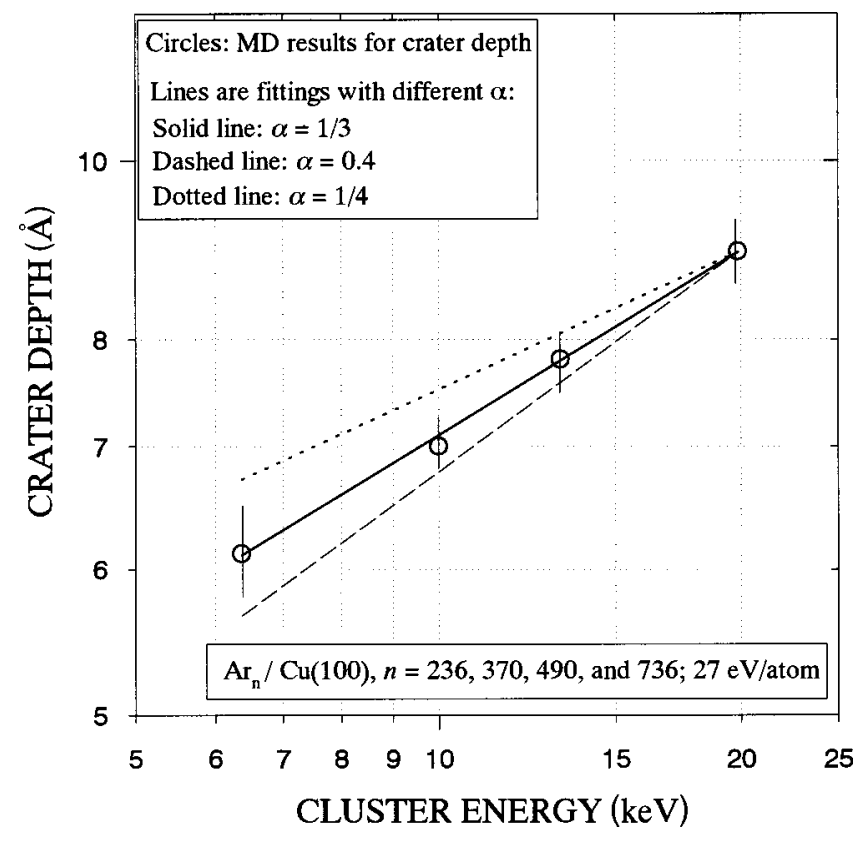

FIG. 3. Crater depth calculated by molecular dynamics for $\operatorname{Ar}_{n}$ $(n=236,370,490$, and 736) cluster ion impact with energy of 27 eV/atom on a $\mathrm{Cu}(100)$ surface (circles), the straight line corresponds to the $E^{1 / 3}$ power law. For comparison, the power-law dependencies with the exponents $\alpha=0.4$ (dotted line) and 0.25 (dashed line) are also given.

components: $P_{c}=21.6 \mathrm{GPa}$ and $P_{\text {th }}=3.35 \mathrm{GPa} .{ }^{37}$ According to this estimation, the internal thermal energy of the compressed material contributing to $E_{i}$ can also be neglected compared with the compression energy of cold material. (In fact, the target area adjacent to a crater may acquire enough thermal energy to be melted, and a rim around a crater can then form by extrusion of the melt, due to plastic flow.)

While the radius of the hemispherical shock front is increasing with time, the mass of the compressed target material increases proportionally to the cube of the radius, which eventually reduces its energy. The radius at which the shock wave stops could be estimated by equating the Hugoniot's pressure $P_{H}$ to the Brinell hardness number of the surface material, meaning that at that radius the shock cannot compress the material anymore. Taking into account the condition $E_{\text {in }} \sim E_{\text {hyd }}$, this defines the Brinell hardness value for the cold pressure from Eq. (2a).

According to the formulas (2), the crater depth is proportional to the $\frac{1}{3}$ power of the total cluster energy $h \sim E_{0}^{1 / 3}$. [This relation ceases to be valid for pressures above $\sim 10^{5} \mathrm{MPa}$, a very rare case for cluster ion impacts with total energies below $300 \mathrm{keV}$ (this estimate for the maximum attainable pressure could be obtained from the energy conservation law as $P_{\max }<E_{0} / V_{\mathrm{cr}}$, and use the crater radius $\sim 100$ $\AA$ from experiment ${ }^{21,22}$ )]. Figure 3 shows the $\frac{1}{3}$ power dependence of the crater depth on the total cluster energy calculated by MD for impacts of $\operatorname{Ar}_{n}(n=236,370,490$, and 736), with energies of $6.4-19.9 \mathrm{keV}$. In order to examine the sensibility of our MD crater depth results to a power law dependence exponent, two other dependencies are plotted in this figure: the dotted line, with $\alpha=\frac{1}{4}$, and the dashed line, with $\alpha=0.4$. As can be seen, the $\frac{1}{3}$ power law is the best fit of these results. 


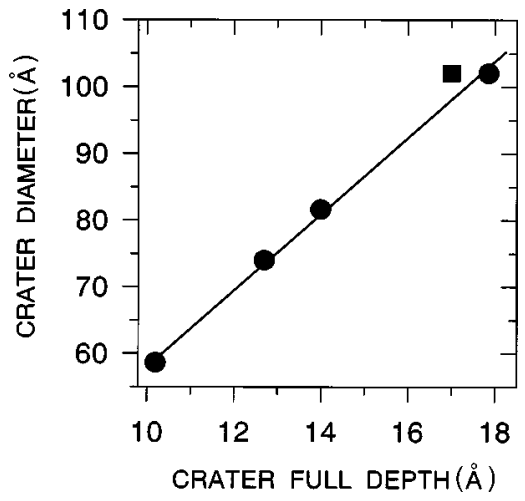

FIG. 4. MD calculation of a diameter $d$ (full) crater depth $h$ dependence. Here, the full crater depth was defined from the top of the rim to the bottom of the crater. Energies and cluster sizes are the same as in Fig. 3.

Excellent linearity between the crater depths and the crater diameters was also obtained in our simulations. Figure 4 shows a linear dependence between the full crater depths, $h$, and diameters $d$ obtained by MD in this paper. The full crater depth was defined as a distance between the top of the rim and the bottom of the crater. The circles in this figure are calculations for a small target of $77000 \mathrm{Cu}$ atoms, and the square for a larger target model of $105000 \mathrm{Cu}$ atoms. This figure shows that the crater diameter could replace the crater

a)

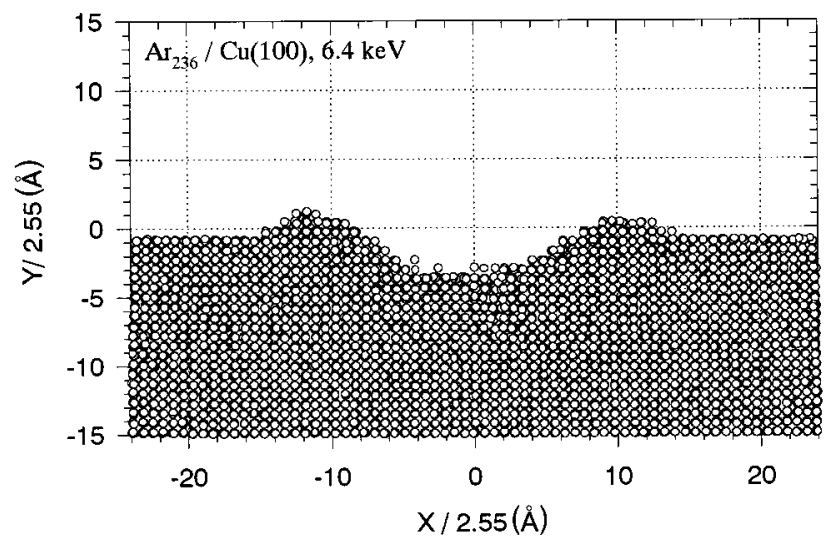

b)

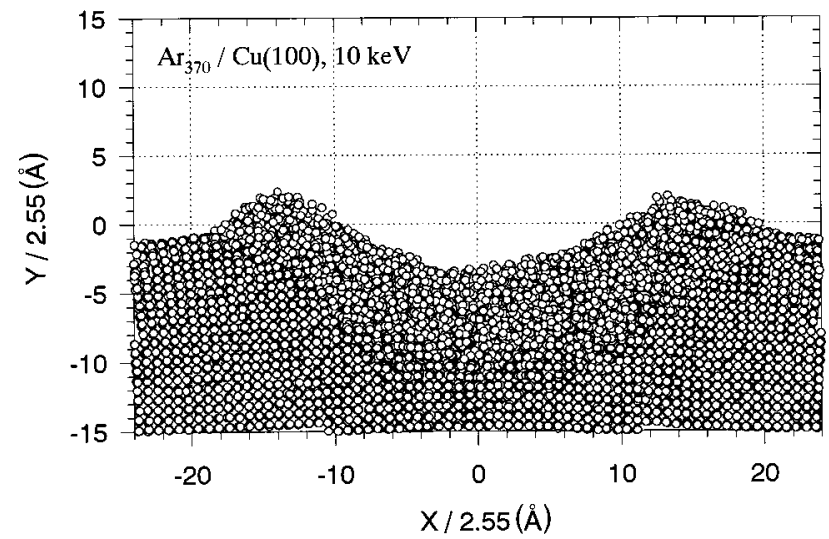

depth, at least for the low energy region. This is important for possible experimental applications as the diameter can be measured with more ease than the depth.

The craters used for the data points in Fig. 3 are shown in Fig. 5 and were obtained by MD for Ar cluster impacts on a $\mathrm{Cu}$ surface, for four cluster sizes given above which correspond to total cluster energies: $6.4 \mathrm{keV}$ (a), $10 \mathrm{keV}$ (b), 13.2 $\mathrm{keV}$ (c), and $19.9 \mathrm{keV}$ (d).

Figure 6 shows measured values of crater diameters produced on gold with an $\mathrm{Ar}_{n}$ ion cluster beam of size $n$ $=3000$, at increasing acceleration voltage. The dependence of the crater dimension on $E_{0}^{1 / 3}$ is evident. (It should be noted that the cluster energy $E_{0}$ is directly proportional to the acceleration voltage $V_{a}$.) The energy range suitable for crater formation experiments varies according to the nature of the cluster and the surface material but would typically be between 20 and $150 \mathrm{keV} .^{21,22}$ Hemispherical craters are formed, which exhibit a linear dependence of the crater volume on the total cluster energy.

The phenomena of cluster shock wave generation, crater formation, and surface sputtering are not possible at low cluster energy, or for small cluster sizes. To form a crater on a surface, the cluster velocity should exceed several sound velocities of the surface material, which gives for Ar cluster energy of about $20 \mathrm{eV} / \mathrm{atom}$. The cluster size should at least be of the order of the shock wave-front thickness which

c)

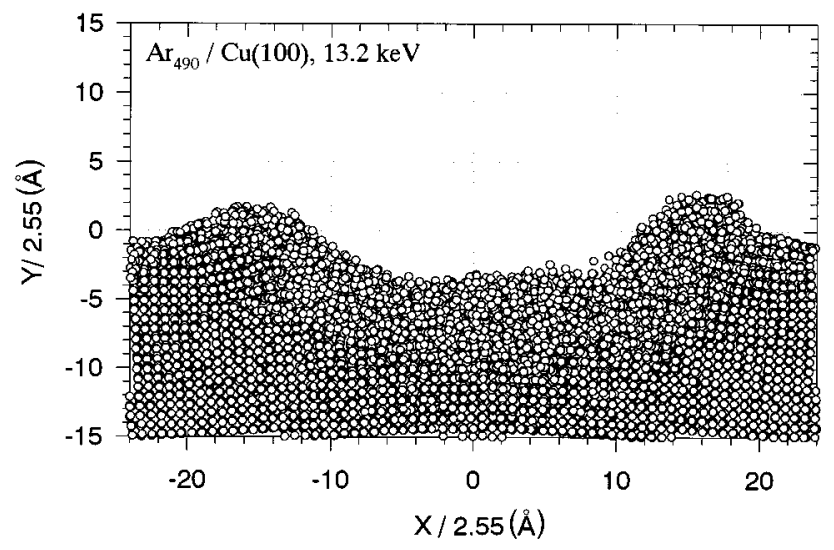

d)

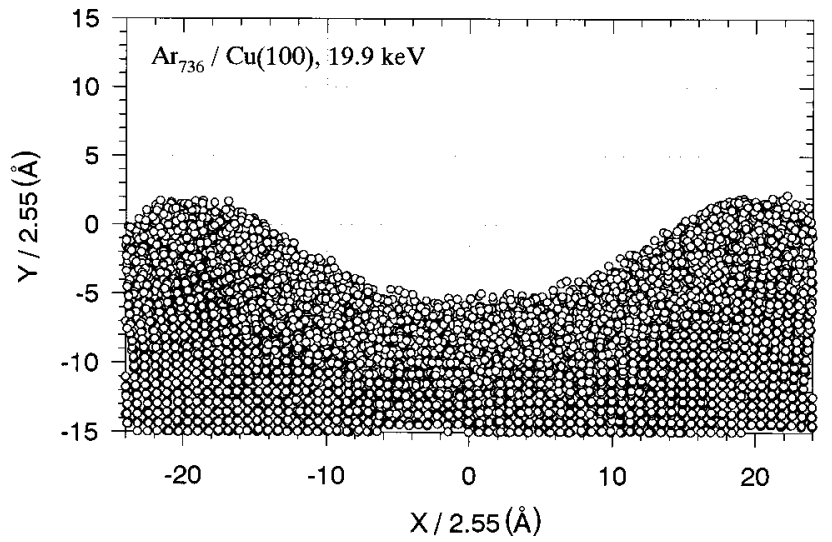

FIG. 5. Side view of the craters formed on a $\mathrm{Cu}(100)$ surface calculated by $\mathrm{MD}$ for $\operatorname{Ar}_{n}(n=236,370,490$, and 736) cluster ion impact, at $6.4 \mathrm{keV}$ after $17 \mathrm{ps}$ (a), and $10 \mathrm{keV}$ (b), $13.2 \mathrm{keV}$ (c), $19.9 \mathrm{keV}$ (d) after 6 ps from the beginning of an impact. 


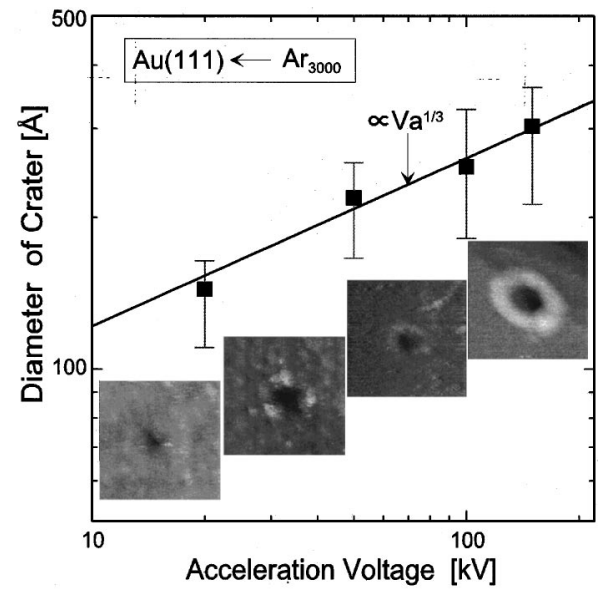

FIG. 6. Energy dependence of the crater diameters obtained by STM (images of the craters are given below each data point) at $\mathrm{Ar}_{3000}$ cluster ion impacts, with total energy of $20-150 \mathrm{keV}$, on a $\mathrm{Au}$ (111) thin film surface deposited on mica (Refs. 21, 22). The straight line in this figure represents the $\frac{1}{3}$ dependence of crater diameters on the acceleration voltage.

could be estimated to be approximately the same as in a planar shock wave, of the order of $50 \AA^{30}$ Therefore, the crater formation phenomenon has thresholds in energy and in cluster size. To complete the discussion on crater formation, it should be also mentioned that the energetic heavy monomer ion can also form a crater on a surface with a probability of about $1 \%,{ }^{11}$ but the physics of crater formation is quite different than that of the cluster case. ${ }^{21,22}$ Such craters, although rare, have occasionally been observed. ${ }^{11}$

\section{SPUTTERING YIELD}

A cluster ion impact is terminated after a time interval of about $\tau \sim d / \nu_{0}$, where $d$ is the cluster diameter and $\nu_{0}$ the cluster velocity. For an Ar cluster of about $10^{3}$ atoms, $d$ $\sim 100 \AA$, and for a cluster energy of about $20 \mathrm{keV}, \nu_{0}$ $\sim 10 \mathrm{~km} / \mathrm{s}$, which gives $\tau \sim 1 \mathrm{ps}$. This estimation agrees with MD simulation results for $\mathrm{Ar}$ cluster impacts on $\mathrm{Au},{ }^{1} \mathrm{Si}^{2}{ }^{2}$ and $\mathrm{Cu}$ (Ref. 39) surfaces. The compressed crater's material forms a rim around the crater by plastic flow, and will be partially sputtered during unloading.

From the definition of binding energy, the total work of a tensile stress which tends to separate target atoms is equal to the binding energy of the target multiplied by a factor which is proportional to the number of atoms removed (i.e., sputtered). This means that for the same energy the lower the binding energy of a material, the higher the sputtering yield. This leads to the conclusion that sputtering yield is most probably linearly proportional to the crater volume. Thus, sputtering yield measurements might also be used as an alternative technique for measuring crater volume.

The crater volume itself depends on the material surface properties, such as sound velocity, compressibility, density, as well as on the cluster properties, such as diameter, type of atoms, etc. It should be noted that the material which is heated above the melting point is removed from the crater and the dynamic response to impact is determined by the mechanical properties of the surrounding area.

The effect of enhanced sputtering on metal, semiconduc- tor and insulator surfaces due to large gas cluster ion bombardment has been experimentally observed ${ }^{1,6}$ and has also been studied by computer simulation. ${ }^{1,39}$ By using MD simulations, we have obtained sputtering yields from $\mathrm{Au}$ and $\mathrm{Cu}$ surfaces due to $\operatorname{Ar}_{n}(n \approx 100-800)$ cluster bombardment at energies of $8-20 \mathrm{keV} .{ }^{39}$ The sputtering yield $Y$ of the material fits a power dependence $Y \sim E^{1.4}$ on the total cluster energy $E$ or, if expressed by the energy per atom, on the cluster size $n . Y$ varies from one material to the other as a function of material properties such as binding energy, sound velocity, etc. The correlation of these effects with Brinell hardness will be discussed in the next section.

\section{BRINELL HARDNESS AND BINDING ENERGY}

At low and intermediate cluster beam energies, $E$ $=5-100 \mathrm{keV}$, a hemispherical crater with a depth $h \sim E^{1 / 3}$ is created on a surface with a single cluster impact. As well, the sputtering rate when the surface is irradiated with many cluster ions is sufficiently high to be significant and measurable.

The crater volume $V_{\mathrm{cr}} \sim h^{3}$, has a linear dependence ( 2 $-b)$ on the total energy divided by the material Brinell hardness

$$
B \sim E_{0} / V_{\text {cr }} .
$$

Thus, the crater depth $h$ has a unique $\frac{1}{3}$ dependence on the cluster energy and on the cold material Brinell hardness, a fact which was previously obtained for hypervelocity macroscopic body impacts on solid (mostly metal) surfaces.

Figures 7(a) and 7(b) present an analysis of available data which confirms these relationships. The correlation between the Brinell hardness number of the target material $-B$, and the energy per sputtered surface atom is represented in Fig. 7(a) and the correlation between the binding energy of the target material per atom and the energy per sputtered atom in Fig. 7(b). The sputtering yield data were taken from Ref. 21. The binding energy and surface hardness data for gold, silver, copper, zirconium, and $\mathrm{SiO}_{2}$ were found elsewhere. ${ }^{13,20,40}$ It is important to note here that $\mathrm{SiO}_{2}$ was included among the data points, which was otherwise obtained for metals. $\mathrm{SiO}_{2}$ is normally used for calibration of hardness measurement equipment and the fact that the relationship for metals is also true for $\mathrm{SiO}_{2}$ should not be underestimated in this case, as it is a good indication that our assumptions are correct.

As mentioned before, crater volume and sputtering yield should correlate with the binding energy of the material of a target. If that is true, a relationship could be obtained between $B$ and energy per sputtered atom, $B$ and binding energy, and between the energy per yield and binding energy. Figure 8 suggests that a linear correlation most probably exists between $B$ and binding energy, but it should be noted here that surface hardness depends also on also on prior treatment of the metal. For example, hardening of a material due to a compressive load (work hardening) should lead to a different crater volume, and to a different sputtering yield. However, to our knowledge, the effect of strain hardening (cold working) on the surface binding energy has not been investigated, and therefore the proposed method should be 
a)

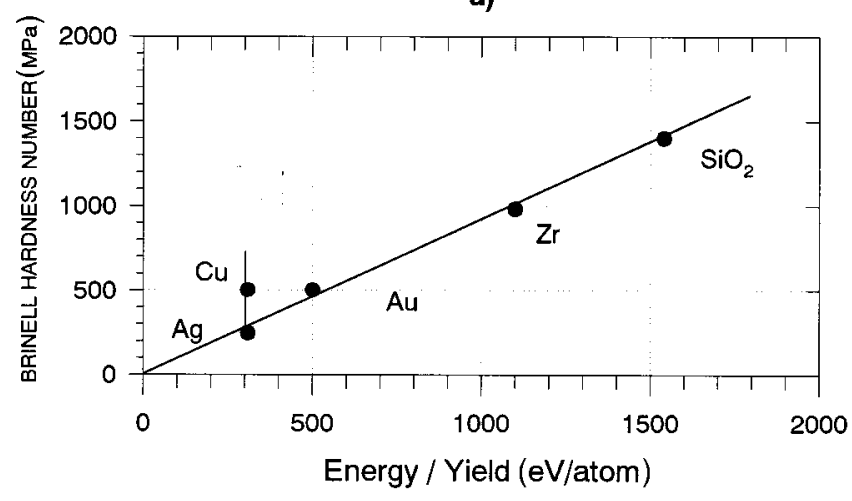

b)

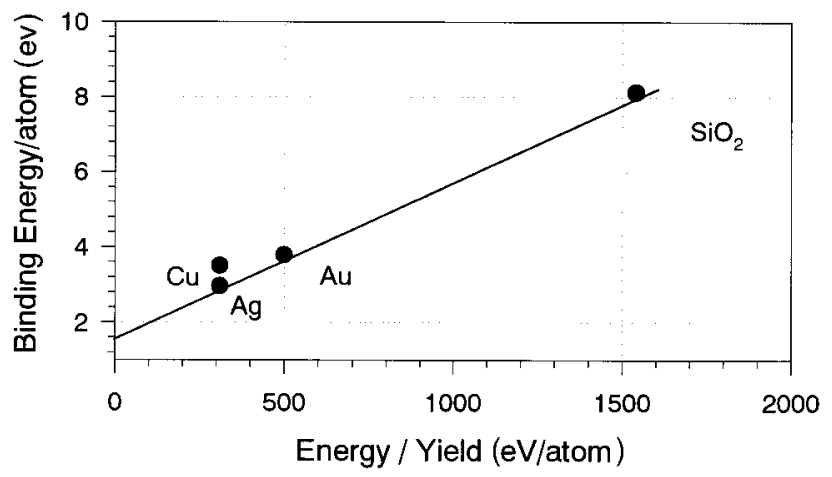

FIG. 7. Brinell hardness data from available literature are correlated linearly to energy per sputtered atom (yield) (a), and binding energy data are correlated with the energy per yield (b). The binding energy for $\mathrm{SiO}_{2} 8.12 \mathrm{eV}$ is taken from Ref. 37.

suitable at this stage for "as-received" or annealed material only. These graphs contain only hardness data for material in its annealed form.

Regarding the stability of crater depth with energy and type of gas, this has been already shown, in both theory ${ }^{2-7}$

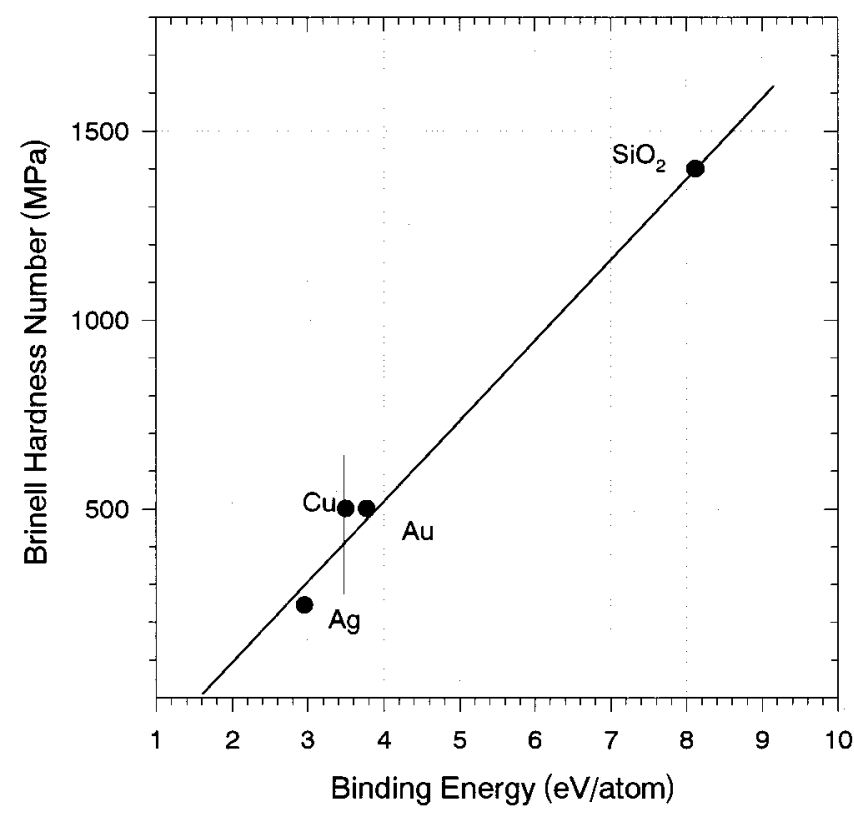

FIG. 8. The feasible linear correlation between the Brinell hardness number, in $\mathrm{MPa}$, and the binding energy, in eV/atom, of the target materials.

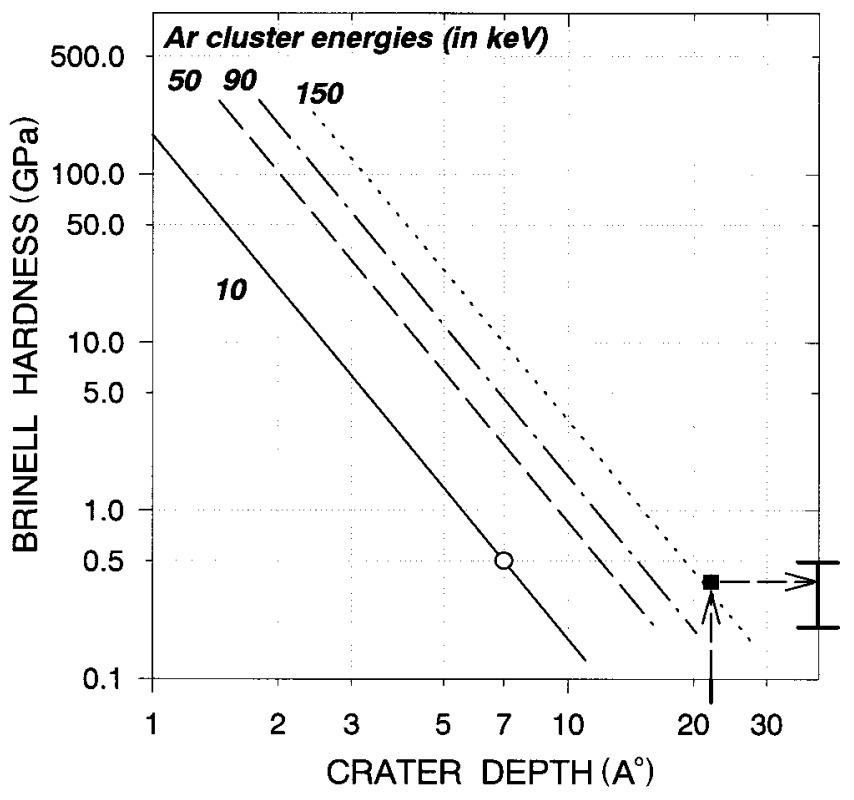

FIG. 9. The Brinell hardness data, in GPa, are correlated with the crater depth, according to formula (3). The total cluster energy is given as a parameter. The calibration of the lines was made for $\mathrm{Cu}$ as target material, for which there are available data for the Brinell hardness, sputtering yield, and binding energy. The circle corresponds to the crater depth of $7 \AA$ obtained by the MD for 10 $\mathrm{keV}$ cluster impact and the Brinell hardness for $\mathrm{Cu}$ from Figs. 6 and 7. The solid line is the calibration line obtained from one data point at $10 \mathrm{keV}$ and with the slope from formula (3). The dash line, dash-dot line, and the dot line were obtained by shifting the solid line according to formula (3). The dashed arrows and the square show our prediction for surface hardness $300 \mathrm{MPa}$ made by using the crater depth of $22 \AA$ formed on a gold (111) surface by irradiation with an $150 \mathrm{keV} \mathrm{Ar}_{3000}$ cluster ion. The error bar on the right axis shows experimental hardness data from literature (Ref. 40).

and experiment, ${ }^{21,22}$ and this data is not shown again in the present paper. Figure 9 shows the double-logarithmic linear dependence between the crater depth $h$ and the hardness $B$ where the total cluster ion energy is given as a parameter. The calibration was made for the calculated data obtained for $\mathrm{Cu}$ sputtering with $\mathrm{Ar}_{370}$ with energy $10 \mathrm{keV}$ (solid line). The crater depth for this impact [cf. with Fig. 5(b)] is about $7 \AA$ and we can use the $\mathrm{Cu}$ hardness of $0.5 \mathrm{GPa}$ from Fig. 7 or 8. The line's slope was found from formula (1). If we use this formula for different cluster energies, we can draw three other lines shown in this figure for $50 \mathrm{keV}$ (dash line), 90 $\mathrm{keV}$ (dash-dot line), and $150 \mathrm{keV}$ (dot line). There is a scarcity of experimental data regarding crater dimensions, as such measurements have rarely been performed. A crater depth of $22 \AA$ was measured in Ref. 22 on a Au (100) surface bombarded with $150 \mathrm{keV} \mathrm{Ar}_{3000}$ cluster ions, and the Brinell hardness of gold is found from the graph as $300 \mathrm{MPa}$ which is within the error bar. ${ }^{40}$ More crater data needs to be collected for materials with well known BHN values so that the slope of the line and the correlation constant could be determined more accurately.

As $d \propto h \propto B^{-1 / 3}$, this obtaining can be used to calibrate various materials by the crater diameter (or depth), and in this way to define a new "true material hardness" scale which can be very useful for example for hard thin film 
coatings deposited on a soft substrate. It is proposed that this finding be used as a new technique for measuring surface hardness. The Brinell hardness $B$, is in fact the cold material pressure $P_{c}$, at which crater is formed, so cluster ion impact is a tool for measuring the cold pressure curve, i.e., it gives a "true hardness" of the material.

This method does not use an indentor as the energy is instantaneously delivered by cluster impact. The damage occurs in subsurface region and is not affected by the substrate. These elements lead to the conclusion that large gas cluster ions are potentially very suitable for use as hardness probes. The technique requires proper calibration and a register of craters needs to be collected for future use as a hardness database. Thus, sputtering yield measurements might also be used as an alternative technique for measuring material hardness.

\section{SUMMARY AND CONCLUSIONS}

The theoretical considerations for a method of hardness measurements using craters produced by cluster ion beam impacts instead of indentor penetration are presented. The cluster impact leaves a hemispherical crater on the surface, which varies in size with surface hardness and cluster parameters (which can be predetermined). This method is based entirely on a surface effect which depends only on the surface material and not on the substrate and therefore is particularly suitable for thin deposited films. This technique also eliminates the need for indentor based measurements. The following was shown.

The Brinell hardness of a material plays a significant role in the evolution of cluster shock waves which agrees with the previous results on the linear dependence of $B$ on the projectile energy per crater volume at macroscopic body impacts on metal surfaces. Atomic scale shock waves generated in a target material and crater formation at accelerated cluster ion impacts were studied by a simplified hydrodynamic theory and molecular dynamics simulations of $\operatorname{Ar}_{n}(n$ $\approx 240-740$ ) cluster ion impacts, with energies of 6.4-19.9 $\mathrm{keV}$, on a $\mathrm{Cu}(100)$ surface. A $\frac{1}{3}$ power-law dependence of the crater depth on the cluster energy was obtained for craters formed on a $\mathrm{Cu}(100)$ surface, at Ar cluster energies of 6.4$19.9 \mathrm{keV}$.

Sputtering yield with cluster ion impacts is suggested to be linearly proportional to crater volume. The cluster energy divided by the sputtering yield (energy per yield) is suggested to be linearly proportional to the Brinell hardness of the material $E_{0} / Y \sim B$, and can also be used for hardness measurements.

With minor changes this feature can be particularly useful for measuring hardness of hard materials such as boron nitride $(\mathrm{BN})$ or $\mathrm{CN}_{x}$ films, as well as a general method for hard thin films on soft substrates. With proper care, this method may also be applicable for materials such as diamond (for which no other technique is available) or alumina, which are affected by ion bombardment, provided very low doses are used.
${ }^{1}$ I. Yamada, W. L. Brown, J. A. Northby, and M. Sosnowski, Nucl. Instrum. Methods Phys. Res. B 99, 223 (1993).

${ }^{2}$ Z. Insepov, M. Sosnowski, and I. Yamada, in Laser and Ion Beam Modification of Materials, edited by I. Yamada et al. (Elsevier, Amsterdam, 1994), p. 111.

${ }^{3}$ I. Yamada, J. Matsuo, Z. Insepov, and M. Akizuki, Nucl. Instrum. Methods Phys. Res. B 106, 165 (1995).

${ }^{4}$ T. Aoki, J. Matsuo, Z. Insepov, and I. Yamada, Nucl. Instrum. Methods Phys. Res. B 121, 49 (1997).

${ }^{5}$ I. Yamada and J. Matsuo, in Advanced Metallization for Future ULSI, MRS Symposia Proceedings No. 427, edited by K. N. Tu, J. W. Q. Mayer, J. M. Poate, and L. J. Chen (Materials Research Society, Pittsburgh, 1996), p. 263.

${ }^{6}$ R. S. Averback and Mai Ghali, Nucl. Instrum. Methods Phys. Res. B 90, 191 (1994).

${ }^{7}$ H. Haberland, Z. Insepov, and M. Moseler, Phys. Rev. B 51, 11061 (1995).

${ }^{8}$ J. K. Dines and J. M. Walsh, in High-Velocity Impact Phenomena, edited by R. Kinslow (Academic, New York, 1970), Chap. 3, p. 45.

${ }^{9}$ J. W. Gehring, in High-Velocity Impact Phenomena (Ref. 8), Chap. 9, p. 463.

${ }^{10}$ D. Maxwell, in Impact and Explosion Cratering, edited by D. J. Roddy, R. O. Pepin, and R. B. Merrill (Pergamon, New York, 1977), p. 1003.

${ }^{11}$ K. L. Merkle and W. Jäger, Philos. Mag. 44, 741 (1981).

${ }^{12}$ D. A. Thompson and S. S. Johar, Appl. Phys. Lett. 34, 342 (1979).
${ }^{13}$ Sputtering by Particle Bombardment I, Physical Sputtering of Single-Element Solids, edited by R. Behrisch (Springer, Berlin, 1981).

${ }^{14}$ J. Gspann, in Physics and Chemistry of Finite Systems: From Clusters to Crystals, Vol. 374 of NATO Advanced Study Institute, Series C: Mathematical and Physical Sciences, edited by P. Jena et al. (Kluwer, Dordrecht, 1992), p. 1115.

${ }^{15}$ D. M. Teter and R. J. Hemley, Science 271, 53 (1996).

${ }^{16}$ D. M. Teter, MRS Bull. 23 (1), 22 (1998).

${ }^{17}$ H. O. O'Neill, The Hardness of Metals and its Measurement (Chapman and Hall, London, 1934).

${ }^{18}$ D. Newey, M. A. Wilkins, and H. M. Pollock, J. Phys. E 15, 119 (1982).

${ }^{19}$ W. D. Callister, Jr., Materials Science and Engineering (Wiley, New York, 1985), pp. 98-103.

${ }^{20}$ R. Manory, Surf. Eng. 3, 233 (1987).

${ }^{21}$ D. Takeuchi, K. Fukushima, J. Matsuo, and I. Yamada, Nucl. Instrum. Methods Phys. Res. B 121, 493 (1997).

${ }^{22}$ T. Seki, T. Kaneko, D. Takeuchi, T. Aoki, J. Matsuo, Z. Insepov, and I. Yamada, Nucl. Instrum. Methods Phys. Res. B 121, 498 (1997).

${ }^{23}$ C. Ronchi, J. Appl. Phys. 44, 3575 (1973).

${ }^{24}$ G. Carter, Radiat. Eff. Lett. Sect. 43, 193 (1979).

${ }^{25}$ K. B. Winterbon, Radiat. Eff. Lett. Sect. 57, 89 (1980).

${ }^{26}$ Y. Kitazoe, N. Hiroaka, and Y. Yamamura, Surf. Sci. 111, 381 (1981).

${ }^{27}$ I. S. Bitenskii and E. S. Parilis, Nucl. Instrum. Methods Phys. Res. B 21, 26 (1987). 
${ }^{28}$ A. Paskin and G. J. Dienes, J. Appl. Phys. 43, 1605 (1972).

${ }^{29}$ V. Yu. Klimenko and A. N. Dremin, Dokl. Akad. Nauk (SSSR) 25, 4379 (1980) [Sov. Phys. Dokl. 25, 288 (1980)].

${ }^{30}$ B. L. Holian, W. G. Hoover, B. Moran, and G. K. Straub, Phys. Rev. A 22, 2798 (1980).

${ }^{31}$ B. L. Holian, in Shock Waves and Spallation by Molecular Dynamics, in Microscopic Simulations of Complex Hydrodynamic Phenomena, Vol. 292 of NATO Advanced Study Institute, Series B: Physics, edited by M. Mareschal and B. L. Holian (Plenum, New York, 1992), p. 75.

${ }^{32}$ R. P. Webb and D. E. Harrison, Jr., Appl. Phys. Lett. 39, 311 (1981).

${ }^{33}$ U. Even, I. Shek, and J. Jortner, Chem. Phys. Lett. 202, 303 (1993).

${ }^{34}$ Z. Insepov and I. Yamada, Nucl. Instrum. Methods Phys. Res. B
112, 16 (1996).

${ }^{35}$ Z. Insepov, M. Sosnowski, and I. Yamada, Nucl. Instrum. Methods Phys. Res. B 127/128, 269 (1997).

${ }^{36}$ A. Rahman, Phys. Rev. A 159, 98 (1967); E. Egami, K. Maeda, and V. Vitek, Philos. Mag. 41, 883 (1980).

${ }^{37}$ Ya. B. Zel'dovich and Yu. P. Raizer, Physics of Shock Waves and High Temperature Hydrodynamic Phenomena (Academic Press, New York, 1967).

${ }^{38}$ L. J. Dhareshwar, P. A. Paik, T. C. Kaushik, and H. C. Pant, Laser Part. Beams 10, 201 (1992).

${ }^{39}$ Z. Insepov, M. Sosnowski, and I. Yamada, Mater. Chem. Phys. 54, 234 (1998).

${ }^{40}$ F. A. McClintock and A. S. Argon, Mechanical Behavior of Materials (MIT, Cambridge, 1964), p. 455. 